\title{
Properties of New York/New Jersey Harbor Sediments
}

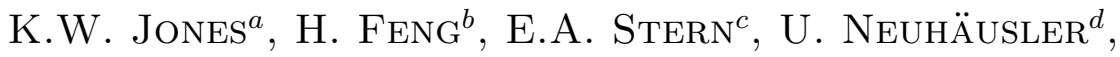 \\ J. OsÁn ${ }^{e}$, N. MARINKOVIC ${ }^{f}$ AND Z. SONG ${ }^{a}$ \\ ${ }^{a}$ Brookhaven National Laboratory, Upton, NY 11973-5000, USA \\ ${ }^{b}$ Montclair State University, Montclair, NJ 07043, USA \\ ${ }^{c}$ U.S. Environmental Protection Agency Region 2 \\ NY, New York 10007-1866, USA \\ ${ }^{d}$ University of Bielefeld, 33501 Bielefeld, Germany \\ ${ }^{e}$ KFKI Atomic Energy Research Institute, 1525 Budapest, Hungary \\ ${ }^{f}$ Albert Einstein College of Medicine, Bronx, NY 10461, USA
}

Sediments found in waterways around the world may contain toxic compounds of anthropogenic origin that can harm the environment and human health. As a result, it is often necessary to remove them and find disposal methods that are environmentally and economically acceptable. Here, we report on results obtained in an experimental program to characterize the nature of the sediment contamination. The objective was to gain a better understanding of the properties of the sediments to develop better methods for understanding the fate and transport of the contaminants and for improving methods for their removal from the sediments. Our investigations made use of X-ray facilities at the Brookhaven National Synchrotron Light Source and the European Synchrotron Radiation Facility at Grenoble, France. The experiments included: measurements of the microstructure of the sediments using computed microtomography, X-ray absorption, and fluorescence microscopy with resolutions as low as 0.2 micrometers to obtain information on the relationships of organic and mineral components of the sediments and on the distribution of contaminants on the surfaces of the sediment grains, investigation of functional groups of chemical compounds using X-ray absorption near-edge spectroscopy and Fourier transform infrared spectroscopy. Scanning electron microscopy and electron probe measurements were made to ascertain the morphology of the sediment surfaces and the distribution of metals on individual sediment grains.

PACS numbers: 89.60.-k, 89.20.--a

\section{Introduction}

Environmentally responsible management of sediments and soils containing potentially toxic organic and inorganic compounds is a problem of worldwide im- 
portance. There is an urgent need to provide affordable methods to remove the contaminants and beneficially use the decontaminated material. The New York $(\mathrm{NY}) /$ New Jersey (NJ) Harbor is a specific example of this broad problem. The Harbor, which is an immense natural resource in the center of a densely populated urban area, is a critical habitat for estuarine and marine life and a stopping place on the Atlantic flyway for migratory birds. It is used for recreational activities by millions and generates important business opportunities based on these recreational uses. The Harbor is also the location of the Port of New York/New Jersey which is the largest port on the eastern seaboard and contributes to the economics of New York, New Jersey, and other nearby states.

However, the Harbor is naturally shallow (approximately $6 \mathrm{~m}$ ) and must be routinely dredged to maintain navigation channels and private berthing facilities that are crucially important for the operation of shipping activities. Sediments found in the Harbor are contaminated with varying levels of organic and inorganic compounds [1]. Some of these contaminants may adversely impact the health of the aquatic environment and thus, in many cases, the sediments are unacceptable for unrestricted placement in an aquatic environment.

Therefore, there is an urgent need for developing other options to dispose each year of the several million cubic yards of dredged material produced in the dredging of federal navigation channels or remediation of specific sediment "hot spots" in the Harbor. The application of sediment decontamination technologies is one component of a potential approach for the management of dredged material in the Port of NY/NJ. The great challenge is to find practical, environmental and economically sound solutions for the management of the dredged material that meet the environmental, commercial, and recreational needs of the Harbor users.

An informed program for dredged material management must integrate information obtained on properties of the sediments covering size scales from nanometers to kilometers. Obviously a great amount of data has accumulated on many of the relevant issues. In our experiments, we have looked at topics that can be effectively investigated using synchrotron radiation sources and other techniques to determine both chemical and physical properties on the submicrometer, micrometer, and other larger-size scales. A summary of some of the experiments done to illuminate several different issues is given here [2].

Our emphasis to date has been on the examination of complex sediments obtained from different locations in the Harbor. We have not attempted to simplify the problem by study of well-defined simple systems, but concentrate on trying to define the actual very complex systems in ways that support understanding of the fate and transport of the contaminants and on how the contaminant materials can be removed.

\section{General properties of the NY/NJ Harbor sediments}

The sediments in the Harbor are heterogeneous, but the surface sediments tend to be a fine-grained mixture of clays, silts, sands, and gravels. Organic 
materials are present in most cases with concentrations around $5 \%$ or more. Toxic organic and inorganic compounds are found in a wide range of concentrations. The distribution of grain sizes in the sediments is given in Fig. 1. This shows that generally $50 \%$ of the sediments have grain sizes less than $10-20 \mu \mathrm{m}$. Concentrations of contaminants found in surface sediments taken from Newtown Creek in New York City $\left(40^{\circ} 43^{\prime} 24.14^{\prime \prime} \mathrm{N}\right.$ and $\left.73^{\circ} 55^{\prime} 28.31^{\prime \prime} \mathrm{W}\right)$ are given in Table. Further measurements were made to estimate settling times of the sediments, mass loss as a function of temperature, and major oxide composition. The results are useful for consideration of methods for decontamination and for creation of beneficial use products following treatment.

TABLE

Contaminants found in Newtown Creek, New York, New York.

\begin{tabular}{l|c|c}
\hline \multicolumn{1}{c|}{ Contaminant } & Newtown Creek $^{a}$ & NY Resid. $^{b}$ \\
\hline $2,3,7,8$ TCDD [ppt] & 9.8 & \\
OCDD [ppt] & 15369 & \\
TCDD/TCDF TEQ [ppt] & 224 & \\
\hline \hline total PCBs [ppm] & 2.86 & 1 \\
\hline \hline anthracene [ppm] & 5820 & 0.5 \\
benzo(a)anthracene [ppb] & 6190 & 224 \\
chrysene [ppb] & 6050 & 400 \\
total PAHs [ppb] & 59380 & 384 \\
\hline \hline total herbicides and DDT [ppb] & 420 & \\
\hline \hline arsenic [ppm] & $5-33$ & 7.5 or $\mathrm{SB}^{c}$ \\
cadmium [ppm] & $1-20$ & 1 or $\mathrm{SB}^{c}$ \\
chromium [ppm] & 305 & 10 or $\mathrm{SB}^{c}$ \\
copper [ppm] & $61-770$ & 25 or $\mathrm{SB}^{c}$ \\
lead [ppm] & $68-554$ & $\mathrm{SB}^{c}$ \\
mercury [ppm] total & $1-3$ & 0.1 \\
nickel [ppm] & $12-140$ & 13 or $\mathrm{SB}^{c}$ \\
silver [ppm] & $2-3$ & $\mathrm{SB}^{c}$ \\
zinc [ppm] & $104-1260$ & 20 or $\mathrm{SB}^{c}$ \\
\hline aA.S. Chen, 1994. Letter Report: Anatical Rest
\end{tabular}

${ }^{a}$ A.S.C. Chen, 1994. Letter Report: Analytical Results of NY/NJ Harbor Sediments. Base-Catalyzed Dechlorination Demonstration Project, Battelle, Columbus, OH. Correspondence to A. Massa, US EPA, Region 2, New York, NY.

${ }^{b} \mathrm{NY}$ Department of Environmental Conservation. Recommended soil cleanup objectives. HWR-94-046 (Revised). January 24, 1994.

${ }^{c} \mathrm{SB}=$ site background. 


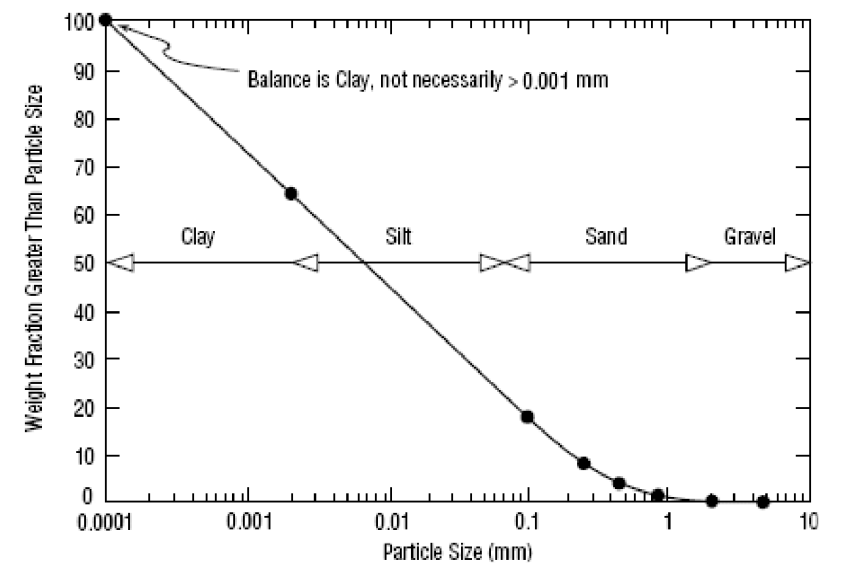

Fig. 1. Grain size distribution found in sediments from the New York/New Jersey Harbor.

\section{Distribution of contaminants on single sediment grains}

We examined a composite sample of sediment particles taken from 6 different locations around the Harbor using an electron microprobe. The samples were sieved to obtain particles with diameters generally less than $10 \mu \mathrm{m}$. Three hundred particles were measured using electron energies of $10 \mathrm{keV}$ and $20 \mathrm{keV}$. Relative concentrations of 20 elements were found: C, N, O, Na, Mg, Al, Si, P, S, Cl, K, $\mathrm{Ca}, \mathrm{Ti}, \mathrm{Cr}, \mathrm{Mn}, \mathrm{Fe}, \mathrm{Ni}, \mathrm{Cu}, \mathrm{Zn}$, and $\mathrm{Pb}$. The values for $\mathrm{Pb}$ are shown in Fig. 2. A

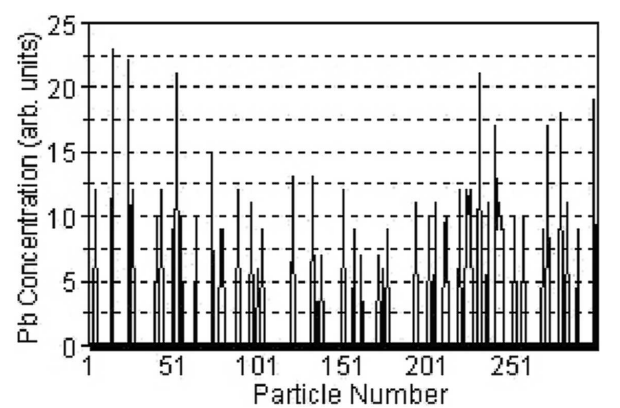

Fig. 2. $\mathrm{Pb}$ concentrations measured on individual sediment grains from NIST SRM 1944, New York/New Jersey Waterway Sediments.

substantial fraction of the sediment grains contain detectable amounts of lead. No obvious correlations with other elements were found through a principal component analysis. Thus, there are no clear insights for choosing methods to use for removal of the lead from the sediment particles.

Investigations of related material from the U.S. National Institute of Standards and Technology Standard Reference Material SRM 1944, New York-New 


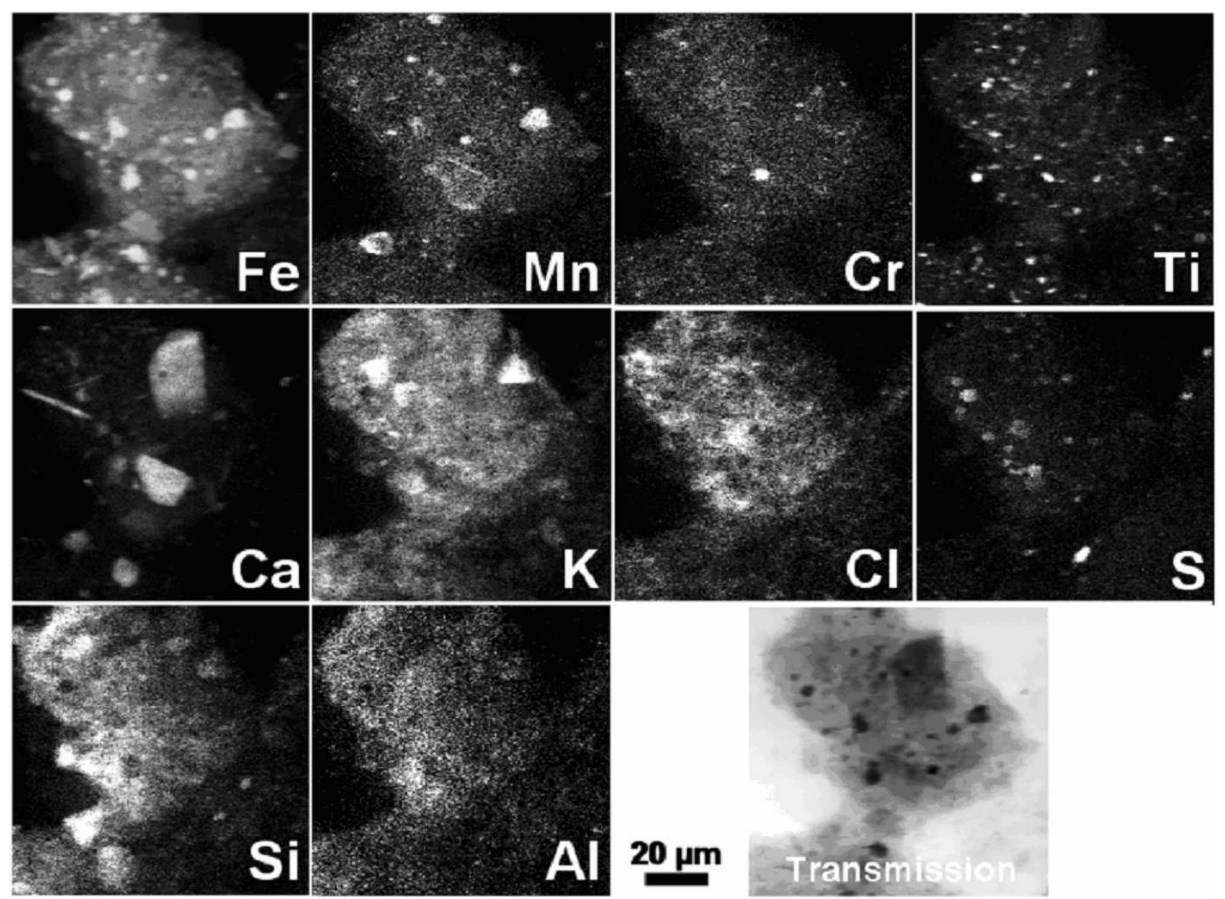

Fig. 3. Maps of trace elements found at ESRF for the NIST SRM 1944 sediments.

Jersey Waterway were carried out at the ID21 micro focus beam line at the European Synchrotron Radiation Facility (ESRF) [3]. X-ray beams with energies of 3 and $8 \mathrm{keV}$ with a spatial size of about $100 \mathrm{~nm}$ were employed. While we have excellent lateral resolution, the depth of material analyzed is of the order of $100 \mathrm{~s}$ of micrometers, depending on the energy of the fluorescent X-rays being detected. Typical data obtained at the $3 \mathrm{keV}$ energy are shown in Fig. 3. The scans showed that many of the elements are found in small regions on larger particles implying that the larger sediment grains may often be associated with smaller particles in the micrometer size range. This result implies that sediment washing technologies could be useful in removing contaminants from sediments if they can breakup the agglomerates.

\section{Measurement of chemical functional groups}

Fourier transform infrared (FTIR) spectroscopy is a powerful method for gaining insights into the nature of the chemical compounds found in the sediments. The U2B beam line at the National Synchrotron Light Source (NSLS) makes possible reflection measurements with spatial resolutions of $14 \mu \mathrm{m}$ with wave numbers ranging from 800 to $3500 \mathrm{~cm}^{-1}$. We investigated sediment particles taken from dredged Harbor sediments at several locations and depths. The wet 


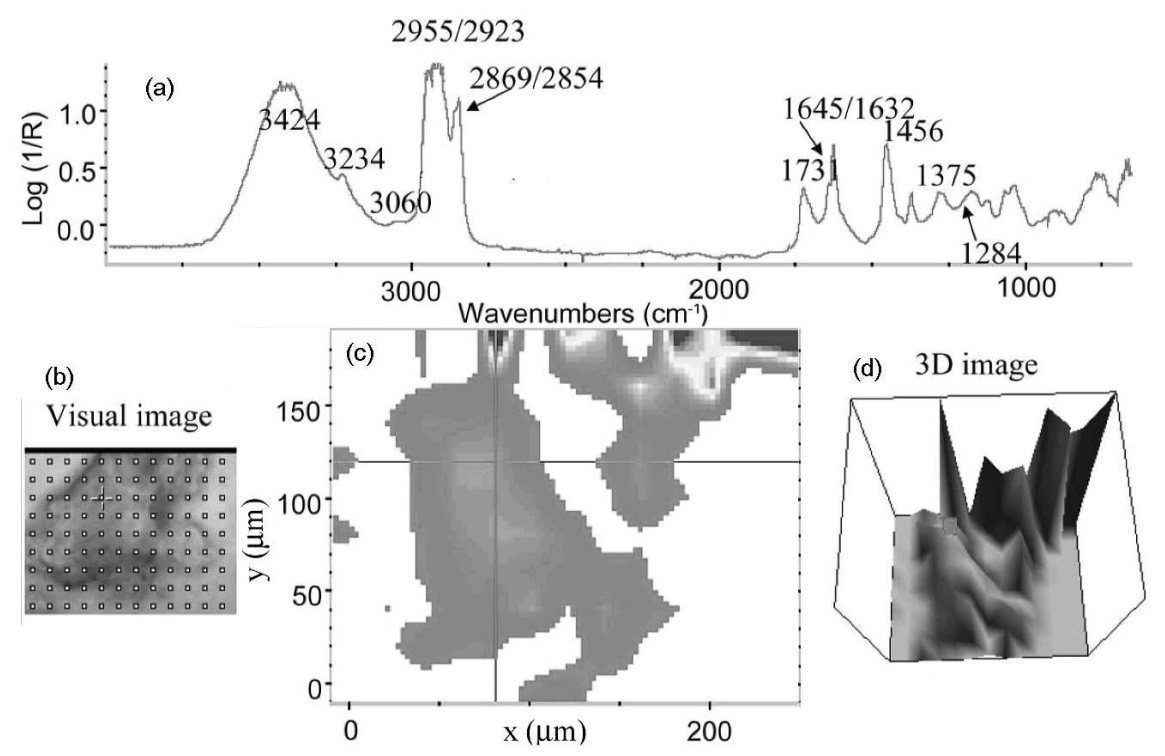

Fig. 4. FTIR data obtained from a methanol extraction from typical Harbor sediment. A typical FTIR spectrum is shown in (a), an optical picture of the sample material is given in part (b), and in (c) and (d) maps of the intensity variation of the $\mathrm{C}-\mathrm{H}$ stretch peaks found in the region from about 2800 to $3000 \mathrm{~cm}^{-1}$. The map (c) is a gray-scale representation of the intensities. The map (d) is a 3-dimensional representation of the data. The strength of the $\mathrm{C}-\mathrm{H}$ stretch peaks can be used as an indicator for the presence of compounds of anthropogenic origin.

sediments were dried onto IR reflecting slides for the measurements. The measurements are restricted to specimens with a thickness in the 10-20 $\mu \mathrm{m}$ range. For this reason, we also extracted organic compounds from the sediment particles using several solvents. Area scans were made over both types of samples. The scans showed that the nature of the organic compounds present were heterogeneous on the $14 \mu \mathrm{m}$ scale for both cases. Comparison of the contaminated sediments with naturally occurring ones shows that the $\mathrm{C}-\mathrm{H}$ stretch bands at 2850 and $2920 \mathrm{~cm}^{-1}$, indicative of aliphatic-type compounds, can be used to obtain an estimate of the importance of anthropogenic compounds in the sample. Data obtained from using an ethanol extract are given in Fig. 4. Spectra varied from point to point in the map showing the heterogeneity mentioned above.

\section{Measurement of sediment microstructure}

The microstructure of the sediments is an important consideration in understanding the transport of contaminants and for developing innovative methods for separation of contaminants from the sediment particles. High-resolution measurements of the structures were made at the NSLS X1A beam line using an environmental cell to contain the wet sediments [4]. 


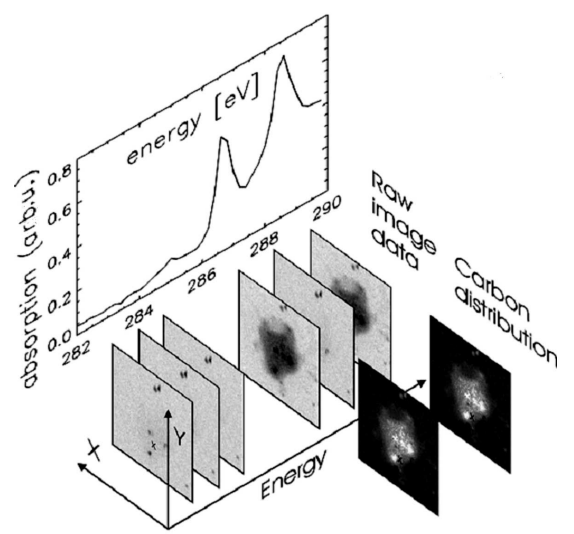

Fig. 5. Maps over several sediment particles contained in an aqueous phase in an environmental wet cell taken at the NSLS X1A beam line. The changes in the maps show the increased carbon absorption above the $\mathrm{C}_{K}$ X-ray absorption edge. The X-ray absorption near-edge structure (XANES) spectrum found at one point is also included.

Radiographs of the specimens were obtained using a raster scan of a $100 \mathrm{~nm}$ beam over a small area of the sample. Scans taken above and below the carbon $K$ absorption edge clearly delineated the carbonaceous material location. Scans over the absorption edge region defined the type of compound present at that point. The results of scans over a sediment particle at different energies are shown in Fig. 5. The maps show that separation of organic and inorganic materials is also useful for micrometer size particles.

Computed microtomography was used to obtain data on the packing of particles in a volume of sediment. We employed an area X-ray detector at the NSLS

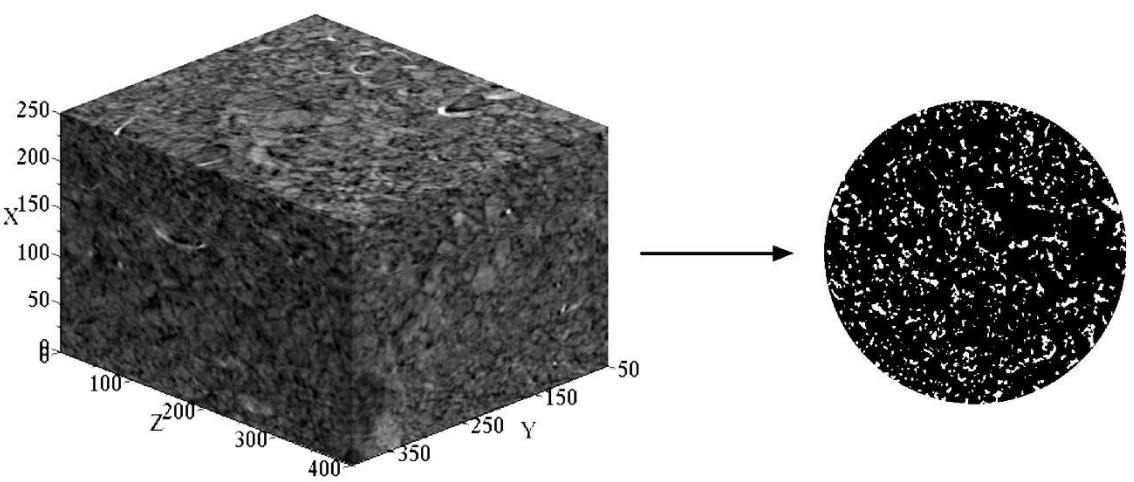

Fig. 6. Three-dimensional volume visualization of sediment particles from Newtown Creek obtained with X-ray computed microtomography at the NSLS X27A beam line. The voxel size is $6.8 \mu \mathrm{m}$. In addition, a slice through the volume is shown after conversion to a binary format showing pore pixels (white) and solid pixels (black). The binary form is used as the starting point for analysis of the microstructure. 
beam line X27A to obtain tomographic data with a voxel size of $2.7 \mu \mathrm{m}$ on a side. A view of a portion of the total volume is shown in Fig. 6. The data are analyzed using the 3Dma-rock program developed by Lindquist et al. [5]. Values for the porosity, permeability, specific surface area, tortuosity, and connecting pathways are obtained. Incorporation of a fluid transport model to show flow patterns through the structure is planned for the future.

\section{Discussion and conclusions}

We have outlined a number of different experimental approaches for gaining a better understanding of the properties of sediments found in the NY/NJ Harbor. The results improve our insights into the heterogeneous distribution of contaminants at a grain-size scale and suggest a pathway for investigation of fluid and contaminant transport through the sediments. Potentially, data of this type will be useful in making decisions about contaminant risk and steps for remediation and for evaluation of technologies used for removal of contaminants.

\section{Acknowledgments}

Work supported by the U.S. Department of Energy under Contract No. DE-AC02-98CH10886 and through Interagency Agreement No. DW89941761-01 between the U.S. Environmental Protection Agency Region 2 and the U.S. Department of Energy.

\section{References}

[1] K.W. Jones, H. Feng, E.A. Stern, J. Lodge, N.L. Clesceri, J. Haz. Mat. 85, 127 (2001).

[2] K.W. Jones, H. Feng, A. Lanzirotti, N. Marinkovic, U. Neuhäusler, C. Riekel, L. Vincze, B. Vekemans, I. Szaloki, Z. Song, in: Proc. Second Int. Conf. on Remediation of Contaminated Sediments, Venice (Italy) 2003, Eds. M. Pellei, A. Porta, CD-ROM, Paper I-06, Battelle Memorial Institute, Columbus, Ohio 2004.

[3] U. Neuhäusler, H. Feng, K. W. Jones, J. Phys. (France) IV 104, 439 (2003).

[4] U. Neuhäusler, S. Abend, G. Ziesmer, D. Schulze, D. Stott, K. Jones, H. Feng, in: Proc. 6th Int. Conf. on X-ray Microscopy (XRM 99), Eds. W. Meyer-Ilse, T. Warwick, American Institute of Physics, New York 2000, p. 323.

[5] The analytical software is described at: http://www.ams.sunysb.edu/ lindquis/3dma/3dma_rock/3dma_rock.html. 